\title{
Evaluation of the Efficacy of Single Anastomosis Sleeve lleal (SASI) Bypass for Patients with Morbid Obesity: a Multicenter Study
}

\author{
Tarek Mahdy ${ }^{1} \cdot$ Sameh Hany Emile ${ }^{1}$ (D) Amr Madyan ${ }^{1} \cdot$ Carl Schou $^{2} \cdot$ Abdulwahid Alwahidi $^{3} \cdot$ Rui Ribeiro $^{4}$. \\ Alaa Sewefy ${ }^{5} \cdot$ Martin Büsing ${ }^{6} \cdot$ Mohammed Al-Haifi $^{7} \cdot$ Emad Salih $^{8} \cdot$ Scott Shikora ${ }^{9}$
}

(C) Springer Science+Business Media, LLC, part of Springer Nature 2019

\begin{abstract}
Background Single anastomosis sleeve ileal (SASI) bypass is a newly introduced bariatric and metabolic procedure. The present multicenter study aimed to evaluate the efficacy of the SASI bypass in the treatment of patients with morbid obesity and the metabolic syndrome.

Methods This is a retrospective, seven-country, multicenter study on patients with morbid obesity who underwent the SASI bypass. Data regarding patients' demographics, body mass index (BMI), percentage of total weight loss (\%TWL), percentage of excess weight loss (\%EWL), and improvement in comorbidities at 12 months postoperatively and postoperative complications were collected.

Results Among 605 patients who underwent the SASI, 54 were excluded and 551 (390; 70.8\% female) were included. At 12 months after the SASI, a significant decrease in the BMI was observed ( $43.2 \pm 12.5$ to $\left.31.2 \pm 9.7 \mathrm{~kg} / \mathrm{m}^{2} ; p<0.0001\right)$. The \% TWL was $27.4 \pm 13.4$ and the \%EWL was $63.9 \pm 29.5$. Among the 279 patients with type 2 diabetes mellitus (T2DM), complete remission was recorded in $234(83.9 \%)$ patients and partial improvement in $43(15.4 \%)$ patients. Eighty-six (36.1\%) patients with hypertension, $104(65 \%)$ patients with hyperlipidemia, $37(57.8 \%)$ patients with sleep apnea, and $70(92.1 \%)$ patients with GERD achieved remission. Fifty-six (10.1\%) complications and $2(0.3 \%)$ mortalities were recorded. Most complications were minor. All patients had 12 months follow-up.

Conclusions The SASI bypass is an effective bariatric and metabolic surgery that achieved satisfactory weight loss and improvement in medical comorbidities, including T2DM, hypertension, sleep apnea, and GERD, with a low complication rate.
\end{abstract}

Sameh Hany Emile

Sameh200@hotmail.com

Tarek Mahdy

tmahdy@yahoo.com

Amr Madyan

profamoora@gmail.com

Carl Schou

cfschou@online.no

Abdulwahid Alwahidi

Abdulwahid66@hotmail.com

Rui Ribeiro

ruijsribeiro@gmail.com

Alaa Sewefy

Sewafy@yahoo.co.uk

Martin Büsing

Martin.buesing@yahoo.de

Mohammed Al-Haifi

Dr_alhaifi@hotmail.com
Emad Salih

Emadtahir73@yahoo.com

Scott Shikora

sshikora@bwh.harvard.edu

1 General Surgery Department, Mansoura Faculty of Medicine, Mansoura University Hospitals, Mansoura University, 60 El-Gomhoria Street Dakahlia, Mansoura 35516, Egypt

2 Aker University Hospital, Oslo, Norway

3 Sharjah University Hospital, Sharjah, United Arab Emirates

4 Clinica De Santo Antonio, Lusiadas, Amadora, Lisbon, Portugal

5 General Surgery Department, Minia Faculty of Medicine, Minia University, Minya, Egypt

6 Klinikum Vest, Dorstenerstr, 151, 45657 Recklinghausen, Germany

7 Sidra Hospital, Riggae Area, Kuwait

8 Atakent Hospital, Istanbul, Turkey

9 Brigham and Women's Hospital, Harvard Medical School, Boston, USA 


\section{Introduction}

Obesity and type 2 diabetes mellitus (T2DM) have become major public health problems [1] and bariatric surgery is now recognized to be the most effective treatment for T2DM in obese patients [2].

Malabsorptive procedures represent a common strategy in bariatric surgery. Most of the malabsorptive bariatric operations employ non-specific malabsorption that leads to potential deficiencies of several nutrients such as iron and folic acid [3]. On the other hand, bariatric procedures that involve digestive tract bypassing may result in atrophy of the mucosa as demonstrated by flattening of the villous surface area and an increase in the mitotic frequency [4].

As the digestive physiology is increasingly under investigation, the interacting neuroendocrine signals that control hunger, satiety, and energy expenditure are better understood. The myriad of potential complications observed with the malabsorptive procedures has driven investigators to develop new, less complicated alternatives [5].

The ideal bariatric procedure does not currently exist. Hypothetically, it would modulate the neuroendocrine control of hunger and satiety without inflicting any harm to important digestive functions unrelated to obesity such as gastrointestinal motility, peristalsis, and enzyme secretion. Based on this concept, in 2003, Santoro et al. [6] introduced a sleeve gastrectomy procedure with transit bipartition (SG p TB). The SG $p$ TB is similar to the duodenal switch (DS) but does not completely exclude the duodenum in order to minimize nutritional complications. The SG p TB amplifies the nutritive stimulation of the distal gut while diminishing the exposure of the proximal bowel to nutrients without excluding the duodenum and jejunum as has been reported in the literature [5-10].

The single anastomosis sleeve ileal (SASI) bypass (Fig. 1) is a simplified modification of the SG p TB that entails a single loop anastomosis rather than Roux-en-Y double anastomosis. It may be assumed that the reduction in the number of intestinal anastomoses may be associated with less anastomotic complications and shorter operative time. However, a randomized trial directly comparing the SASI bypass and the SG p TB is required to substantiate this assumption.

A possible explanation of the mechanism of action of the SASI bypass was devised by Mahdi et al. [11] that patients who undergo the SASI bypass eat less food because they experience early satiety due to a hypothalamic-generated satiety sensation which is caused by the perception of nutrients in the distal bowel [12]. The intense distal gut stimulation reduces proximal bowel activity. The distal gut hormones such as GLP-1 have central satietogenic effects, and they also reduce gastric emptying which is known as the ileal break mechanism [13].
The first report on the efficacy of the SASI bypass in patients with morbid obesity demonstrated excellent results of the new procedure [11]. The present multicenter study aimed to evaluate the short-term efficacy of the SASI bypass in the treatment of patients with morbid obesity and the metabolic syndrome in terms of weight loss and remission of T2DM.

\section{Patients and Methods}

\section{Study Design}

This is a retrospective, multicenter study of patients with morbid obesity with or without T2DM who underwent the SASI bypass in the period of January 2014 to January 2018. Prospective data were collected from eight centers located in seven countries $(\mathrm{UAE}=37$, Kuwait $=36$, Egypt $=275$, Germany $=63$, Norway $=71$, Portugal $=19$, and Turkey $=$ 50). Central ethical approval for the study was obtained by the principal investigator from the Research Ethics Committee (code=MOHAP/DXB-REC//MAA/No.15/2019).

\section{Procedures Implemented to Maintain Confidentiality of Participant's Data}

Only the research team was eligible to access the data which was kept anonymous. Responses to the research were reported in aggregated form to protect the identity of respondents. For the purpose of confidentiality, no names of patients were mentioned in the manuscript.

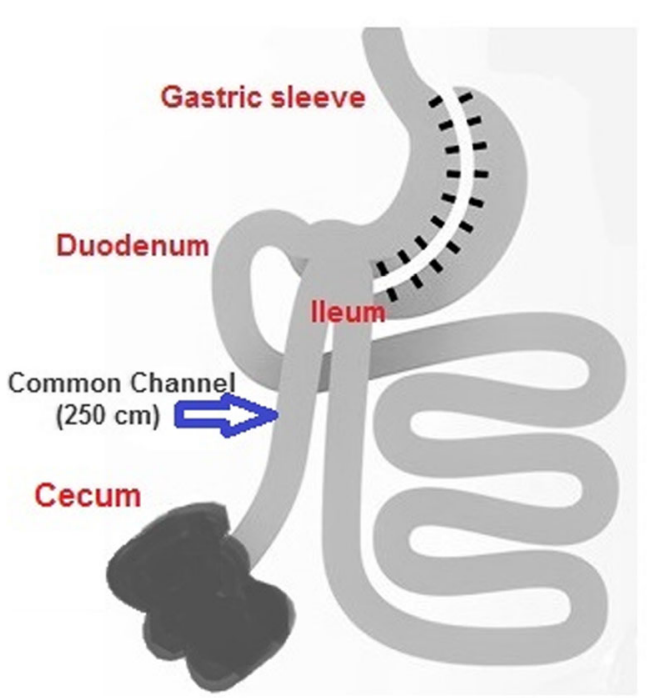

Fig. 1 Schematic demonstration of single anastomosis sleeve ileal (SASI) bypass 


\section{Subject Selection}

Adult patients (18-60 years) of both sexes with morbid obesity were included. Morbid obesity was defined as a body mass index (BMI) of greater than $40 \mathrm{~kg} / \mathrm{m}^{2}$ or greater than $35 \mathrm{~kg} / \mathrm{m}^{2}$ with at least one associated comorbidity. We excluded patients with a previous upper abdominal laparotomy or psychological instability. Patients who did not fulfill at least 12 months of follow-up were also excluded from the study.

\section{Study Outcome Measures}

The primary outcomes of the study were as follows:

- The percentage of total weight loss (\%TWL): calculated as [(preoperative weight - weight at twelve months)/preoperative weight] $\times 100$.

- The percentage of excess weight loss (\%EWL): calculated as: [(preoperative weight - weight at twelve months)/preoperative excess weight] $\times 100$.

- Complete remission of T2DM was defined as a fasting plasma glucose level $<100 \mathrm{mg} / \mathrm{dl}$ or HbA1C level $<6 \%$ without the use of hypoglycemic medication at 1 year after surgery.

- A partial improvement in T2DM was defined as a reduction of at least $25 \%$ of the fasting plasma glucose level and of at least $1 \%$ in the hemoglobin A1c level with the use of hypoglycemic medications [14].

Secondary outcomes were as follows:

- Remission of hypertension was considered if the disease was controlled and the patients was normotensive (BP $<$ 120/80) off antihypertensive medication [14].

- Remission of hyperlipidemia was defined as normal lipid profile off medications [14].

- Remission of sleep apnea was defined as AHI/RDI of less than five off CPAP/BI-PAP on repeat objective testing with polysomnography [14].

- Remission of GERD was defined as absence of symptoms, no medication use, and normal 24-h pH study [14].

- Postoperative complications were defined as "an undesirable and unintended result of the operation affecting the patient that occurs as a direct result of the operation."

- Changes in nutritional status.

\section{Preoperative Assessment and Preparation}

All patients had a preoperative evaluation including careful history taking, clinical examination, and laboratory investigation including blood glucose, lipid profile, and a thyroid and cortisol hormonal evaluation. The diagnosis of T2DM was based on fasting blood glucose concentrations $>126 \mathrm{mg} / \mathrm{dl}$ or those patients with a positive history of diabetes and taking antidiabetic medications.

For each of the obesity-related comorbidities, the following tests were used for diagnosis: T2DM: Fasting and postprandial blood glucose and HBA1c; hypertension: arterial blood pressure measurement on three different occasions under resting conditions; dyslipidemia: serum triglyceride, cholesterol, LDL, VLDL, and HDL level; sleep apnea: STOP-BANG questionnaire and sleep study (polysomnography); and GERD: endoscopy and 24-hour pH study.

Routine gastroscopy or gastrografin studies were also performed. Abdominal ultrasounds were done to exclude gallstones and to evaluate the degree of fatty liver. The liver size was reduced by keeping all patients on a low-calorie protein diet for 2 weeks prior to surgery. Deep vein thrombosis prophylaxis started $12 \mathrm{~h}$ before surgery with low molecular weight heparin subcutaneous injections.

\section{Surgery}

The patient is placed on the operating table in the French position. General anesthesia with endotracheal intubation is performed. The first part of the operation is performed with the patient in a steep reverse-Trendelenburg position and the surgeon standing between the legs of the patient.

The technique commences with the devascularization of the greater curvature of the stomach. The dissection then is continued toward the gastroesophageal junction. The left crus is then completely freed of any attachments to avoid leaving a posterior pouch when constructing the sleeve. Posterior attachments between the stomach and pancreas are then divided.

The stomach is then tabularized over a 36-French calibration tube, with a linear cutting stapler, commencing $6 \mathrm{~cm}$ proximal to the pylorus. The staple line is then oversewn with a running suture. The table is then changed to the horizontal position and the surgeon moves to the left-hand side of the patient to perform the second part of the operation.

The ileocecal junction is identified and a point $250 \mathrm{~cm}$ proximal to the ileocecal valve is measured. The selected intestinal loop is then brought up to the gastric sleeve without division of the greater omentum. A stapled isoperistaltic sideto-side anastomosis to the anterior wall of the antrum of the stomach is done using a linear cutting stapler, $6 \mathrm{~cm}$ proximal to the pylorus. The diameter of the anastomosis should not exceed $3 \mathrm{~cm}$ in diameter. The anterior wall of the gastroileal anastomosis is closed with a two-layer running suture. A methylene blue test is the performed to assess for leaks.

Ambulation and clear liquids are started on the night of surgery. Thrombosis prophylaxis is continued for 4 weeks. Proton pump inhibitors are administrated for 4 months postoperatively. 


\section{Follow-up}

The study patients were seen as outpatients 2 weeks postoperatively then every month for 12 months. Patients were also seen in the clinic if they developed symptoms between their follow-up visits. The endpoint of the study was at 1 year after SASI bypass. When patients reached this time point, they were contacted by email or telephone to visit the clinic for follow-up in terms of weight loss and improvement in comorbidities. Patients who failed to respond at this time point were excluded from the study.

Study patients were placed on a low-caloric, protein-rich liquid diet for the first month and then other elements were sequentially introduced under strict dietitian supervision. The patients were asked to take multivitamin supplements that included a minimum of $18 \mathrm{mg}$ of iron, $400 \mathrm{mcg}$ of folic acid, 800 to $1,000 \mathrm{IUs}$ of vitamin $\mathrm{D}$, and $500 \mathrm{mcg}$ of vitamin $\mathrm{B}_{12}$ on daily basis. The supplement also included calcium, selenium, copper, and zinc. Patients were encouraged to initiate physical activity after the first postoperative week. All patients had a complete blood investigation every 3 months and gastroscopy every 6 months.

\section{Assessments}

At 12 months of follow-up, patient weights were measured. The \% TWL, \%EWL, and BMI were calculated. Remission or partial improvement in T2DM and other comorbidities were recorded. Remission of T2DM was assessed by means of clinical and laboratory parameters; the clinical parameters included reduction of the dose or stoppage of insulin or hypoglycemic medications with normally maintained blood glucose levels. The laboratory parameters were fasting blood glucose $<100 \mathrm{mg} / \mathrm{dl}$ and HBA1c $<6 \%$.

The fasting blood sugar level, HbAlc, serum albumin, serum hemoglobin, serum iron, and serum vitamin $\mathrm{D}$ were measured and compared with their baseline values. Early and delayed procedure-related complications were also recorded.

\section{Data Collected}

For each participating center in this study, there was one person (one of the authors) who performed the SASI bypass and carried out the data collection. Patient and outcome data were stored in an electronic registry. The data were checked for accuracy and were verified before their collection and extraction into Excel sheets. Finally, all authors sent their data in an Excel sheet format to the first author (T.M.). The data management team (second and third authors) conducted a second check of the data for completeness and accuracy. Patients with missing or incorrect data were excluded from the study.

Preoperative data including patient age, gender, initial weight, BMI, excess body weight, and obesity-related comorbidities such as T2DM, hypertension, cardiac ischemia, hyperlipidemia, obstructive sleep apnea syndrome (OSAS), the presence of gallstones, joint pain, depression, infertility, and heartburn were recorded.

Operative data were recorded including intraoperative complications such as bleeding, organ injury, specimen retrieval problems, and stapler malfunction. Postoperative data collection included \%TWL, \%EWL, change in BMI, and early postoperative complications that occurred during the first month such as infections, bleeding, vomiting, leak, and port site problems. Long-term complications beyond 1 month after surgery such as vomiting, reflux, stricture, intestinal obstruction, hypoalbuminemia, anemia, iron, and vitamin $\mathrm{D}$ deficiency were also collected.

\section{Statistical Analysis}

Data were analyzed using IBM $®$ SPSS ${ }^{\circledR}$ (version 21.0 for Windows). Unless stated otherwise, all data were expressed as the mean \pm standard deviation (SD) or as percentages. Descriptive and inferential statistical analyzes were performed using both parametric and non-parametric procedures as appropriate. Comparisons of categorical/ordinal variables were performed using chi-square analysis for trends. Continuous variables were compared using student $t$ test. Multiple linear regression analysis was performed to determine the significant independent predictors for higher \% TWL after SASI bypass. All tests were two-tailed, and the results with $p<0.05$ were considered statistically significant.

\section{Results}

\section{Patients' Characteristics}

After screening the records of 605 patients who underwent the SASI bypass in the study period, 54 did not meet the inclusion criteria of the study or had missing data, thus were excluded. Ultimately, 551 patients from eight participating centers were included in the study. Three hundred ninety patients were female (70.8\%) and $161(29.2 \%)$ were male. The mean age of the patients was $39.1 \pm 14.7$ (range, 18-60) years. The mean preoperative BMI was $43.2 \pm 12.5$ (range, 35-80) kg/ $\mathrm{m}^{2}$. The mean preoperative weight was $119.3 \pm 37.9$ (range, 73.6-234) $\mathrm{kg}$ and the mean preoperative height was $165.2 \pm$ 8.5 (range, 144-193) $\mathrm{cm}$.

A total of 279 patients had T2DM $(77.7 \%$ were on insulin and $22.3 \%$ were on oral antidiabetic medications), 238 had hypertension, 160 had hyperlipidemia, 64 had OSAS, and 76 had gastroesophageal reflux disease (GERD). Fifty-eight $(10.5 \%)$ of the patients underwent the SASI bypass as a rescue procedure after a failed sleeve gastrectomy. The characteristics of the study patients are shown in Table 1 . 
Table 1 Preoperative characteristics of the patients studied

\begin{tabular}{ll}
\hline Variable & Value \\
\hline Number & 551 \\
Female/male & $390 / 161$ \\
Mean age in years & $39.1 \pm 14.7$ \\
Mean body mass index in $\mathrm{kg} / \mathrm{m}^{2}$ & $43.2 \pm 12.5$ \\
Mean weight in $\mathrm{kg}$ & $119.3 \pm 37.9$ \\
Mean height in cm & $165.2 \pm 8.5$ \\
Diabetes mellitus & $279(50.6 \%)$ \\
Hypertension & $238(43.2 \%)$ \\
Hyperlipidemia & $160(29 \%)$ \\
Sleep apnea & $64(11.6 \%)$ \\
Gastroesophageal reflux disease & $76(13.8 \%)$ \\
Previous sleeve gastrectomy & $58(10.5 \%)$ \\
\hline
\end{tabular}

\section{Weight Loss at 12 Months}

The \%TWL was $27.4 \pm 13.4$ (range, 9-56) and the \%EWL was $63.9 \pm 29.5$ (range, 24.5-98.8). At 12 months after the SASI bypass, a significant decrease in BMI was observed (from $43.2 \pm 12.5$ to $31.2 \pm 9.7 \mathrm{~kg} / \mathrm{m}^{2} ; p<0.0001$ ). Similarly, preoperative body weight was also significantly decreased at 12 months of follow-up (from $119.3 \pm 37.9$ to 86.4 $\pm 29.6 \mathrm{~kg}$ ) (Table 2).

\section{Improvement in Comorbidities}

Among 279 patients with T2DM, complete remission was recorded in $234(83.9 \%)$ patients and partial improvement in $43(15.4 \%)$ patients. Therefore, a total of 277 (99.3\%) patients showed either complete remission or partial improvement in their glycemic state after SASI bypass.

Patients who developed complete remission of T2DM showed a significant decrease in fasting blood glucose $(228.4 \pm 103.4$ to $100.4 \pm 16.1 \mathrm{mg} / \mathrm{dl}, p<0.0001)$ and $\mathrm{a}$ significant decrease in HbA1c $(8.1 \pm 3.6$ to $5.3 \pm 2.6 ; p<$ $0.0001)$. Serum C-peptide level increased significantly from $1.37 \pm 0.8$ to $1.6 \pm 1.3 \mathrm{ng} / \mathrm{ml}(p=0.02)$.

Similarly, patients who showed partial improvement in the glycemic state showed significant decrease in fasting blood glucose $(186.3 \pm 73.1$ to $120.2 \pm 43.8 \mathrm{mg} / \mathrm{dl}, p<0.0001)$ and a significant decrease in HbA1c $(7.6 \pm 3.5$ to $5.6 \pm 2.6$; $p=0.003$ ). Serum C-peptide level increased from $0.65 \pm 0.17$ to $0.69 \pm 0.35 \mathrm{ng} / \mathrm{ml}(p=0.5)$.

Eighty-six (36.1\%) of 238 patients with hypertension, 104 $(65 \%)$ of 160 patients with hyperlipidemia, 37 (57.8\%) of 64 patients with OSAS, and $70(92.1 \%)$ of 76 patients with GERD showed remission after having the SASI bypass. Improvements in obesity-related comorbidities were all statistically significant as shown in Table 3.
Table 2 Weight loss at 12 months after SASI bypass

\begin{tabular}{llll}
\hline Variable & Preoperative & Postoperative & $P$ value* \\
\hline Mean body mass index & $43.2 \pm 12.5$ & $31.1 \pm 9.7$ & $<0.0001$ \\
Mean body weight in kg & $119.3 \pm 37.9$ & $86.2 \pm 29.7$ & $<0.0001$ \\
\%Total weight loss & ----- & $27.4 \pm 13.4$ & ---- \\
\%Excess weight loss & ----- & $63.9 \pm 29.5$ & ---- \\
\hline
\end{tabular}

*Unpaired Student $t$ test was used for data analysis

\section{Changes in the Nutritional Status After SASI Bypass}

Changes in laboratory parameters reflecting the nutritional status at 12 months after SASI bypass included a nonsignificant increase in hemoglobin levels $(p=0.23)$, a significant decrease in serum iron levels $(p=0.02)$, a significant decrease in serum albumin levels $(p=0.007)$, and a significant increase in vitamin D levels $(p<0.0001)$. Despite the decrease in serum albumin levels, none of the patients had protein malabsorption postoperatively and the average serum albumin level after SASI was within normal range $(3.9 \mathrm{~g} / \mathrm{dl})$ (Table 4).

\section{Complications and Readmission}

There were fifty-six $(10.1 \%)$ complications after the SASI bypass. Four $(0.72 \%)$ patients required readmission within 30 days after surgery. Postoperative morbidities included bilious vomiting ( $n=32)$, diarrhea $(n=15)$, stomal ulcer $(n=3)$, calcular obstructive jaundice $(n=2)$, pulmonary embolism $(n$ $=1)$, intestinal obstruction $(n=1)$, staple line bleeding $(n=1)$, and ileal perforation $(n=1)$.

Bilious vomiting and diarrhea were treated conservatively with fluids and medications. Stomal ulcers were managed with proton pump inhibitors, and calcular obstructive jaundice was treated with ERCP and stone extraction, whereas staple line bleeding, intestinal obstruction, and ileal perforation required surgical intervention. One patient who developed a pulmonary embolism was admitted to the ICU and was treated with intravenous fluids, anticoagulant medications, and thrombolytic therapy.

Overall, according to the Clavien-Dindo classification, there were $47(84 \%)$ grade I complications, three $(5.3 \%)$ grade II complications, five ( $8.9 \%)$ grade III complications, and one (1.7\%) grade IV complication (Table 5).

\section{Outcome of the SASI Bypass as a Rescue Procedure After Sleeve Gastrectomy}

Fifty-eight patients underwent the SASI bypass as a rescue surgery after sleeve gastrectomy. Patients were 35 (57.6\%) female and $23(42.4 \%)$ male. The mean age of these patients was $41.8 \pm$ 10.5 years. The mean preoperative height was $162 \pm 6.9 \mathrm{~cm}$. Ten (17.2\%) patients had T2DM, 24 (41.4\%) had hypertension, three $(5.1 \%)$ had dyslipidemia, and three $(5.1 \%)$ had OSAS. 
Table 3 Improvement in obesityrelated comorbidities at 12 months after SASI bypass

\begin{tabular}{llll}
\hline Variable & Preoperative & Postoperative & $P$ value* \\
\hline Type 2 diabetes mellitus & 279 & 2 & $<0.0001$ \\
Hypertension & 238 & 152 & $<0.0001$ \\
Hyperlipidemia & 160 & 56 & $<0.0001$ \\
Obstructive sleep apnea syndrome & 64 & 27 & $<0.0001$ \\
Gastroesophageal reflux disease & 76 & 6 & $<0.0001$ \\
\hline
\end{tabular}

The italicized enteries reflect a significant $p$ value $<0.05$

*Chi-square test was used for data analysis
The mean preoperative weight decreased significantly at 12 months after SASI from $109.8 \pm 22.8$ to $90.5 \pm 20.3 \mathrm{~kg}(p<$ $0.0001)$. The mean preoperative BMI decreased significantly at 12 months from $41.1 \pm 5.4$ to $33.5 \pm 5.2 \mathrm{~kg} / \mathrm{m}^{2}(p<0.0001)$. The mean \%TWL at 12 months postoperatively was $17.3 \pm$ 9.3 and the mean $\%$ EWL was $40.9 \pm 22.1$.

All diabetic patients showed complete remission of T2DM, four (16.6\%) patients with hypertension had complete remission, while none of the patients with dyslipidemia or OSAS showed remission of their comorbidity. Seven patients developed bilious vomiting after the SASI bypass. There were no patient mortalities.

\section{Multiple Linear Regression Analysis of \%TWL}

Using the \%TWL as the dependent variable, multiple linear regression analysis revealed that the variables that were significant independent predictors for higher \% TWL were as follows:

- Lower preoperative BMI (estimate $=0.052, \mathrm{SE}=0.007, t$ $=7.2, p=0.0001)$

- Lower preoperative body weight (estimate $=-0.017, \mathrm{SE}$ $=0.003, t=-6.6, p=0.0001)$

- Greater height (estimate $=2.39, \mathrm{SE}=0.38, t=6.33, p=$ 0.0001)

- Performing the SASI bypass as a primary (not a rescue) surgery (estimate $=-0.11, \mathrm{SE}=0.012, t=-8.8, p=$ $0.0001)$

Table 4 Changes in laboratory parameters at 12 months after SASI bypass
On the other hand, the patient's age (estimate $=-5.7, \mathrm{SE}=$ $0.001, t=-0.01, p=0.99$ ) and gender (estimate $=0.017, \mathrm{SE}=$ $0.014, t=1.23, p=0.22$ ) were not significantly associated with higher $\%$ TWL.

\section{Discussion}

As bariatric surgery now comprises a myriad of different procedures, the selection of each bariatric procedure can be a difficult decision. The SASI bypass has the advantage of combining mechanical restriction of the gastric capacity with neuromodulation as an additional strategy to promote the metabolic effects of the procedure. The remarkable impact of the SASI bypass on the glycemic control in diabetic patients is a major advantage of this procedure with remission rates reaching up to $100 \%$. This finding would suggest that the SASI bypass is an excellent option for patients with obesity and T2DM. As the procedure entails only a single gastroileal anastomosis, the risk of anastomotic complications may theoretically be less than other procedures that include more than one anastomosis.

The SASI bypass is a modification of the sleeve gastrectomy by adding a single anastomosis between the gastric antrum and the ileum. While sleeve gastrectomy has become the most common bariatric procedure in many centers owing to its excellent results [15], a number of drawbacks of the procedure have been recognized. These drawbacks include being less effective in super obese patients, a high incidence of postoperative GERD, and weight regain on long-term follow-up $[16,17]$.

\begin{tabular}{llll}
\hline Variable & Preoperative & Postoperative & $P$ value* \\
\hline Mean fasting serum glucose $(\mathrm{mg} / \mathrm{dl})$ & $228.4 \pm 103.4$ & $100.4 \pm 16.1$ & $<0.0001$ \\
Mean HbA1c $(\%)$ & $8.1 \pm 3.6$ & $5.3 \pm 2.6$ & $<0.0001$ \\
Mean serum C-peptide $(\mathrm{ng} / \mathrm{ml})$ & $1.37 \pm 0.8$ & $1.6 \pm 1.3$ & 0.02 \\
Mean hemoglobin $(\mathrm{gm} / \mathrm{dl})$ & $12.7 \pm 5.2$ & $13.3 \pm 10.5$ & 0.23 \\
Mean serum iron $(\mu \mathrm{g} / \mathrm{d})$ & $61.3 \pm 26.9$ & $57.5 \pm 27.3$ & 0.02 \\
Mean serum albumin $(\mathrm{gm} / \mathrm{dl})$ & $4.2 \pm 1.9$ & $3.9 \pm 1.8$ & 0.007 \\
Mean vitamin D $(\mathrm{ng} / \mathrm{ml})$ & $22.4 \pm 18.1$ & $44.2 \pm 33$ & $<0.0001$ \\
\hline
\end{tabular}

The italicized enteries reflect a significant $p$ value $<0.05$

*Unpaired Student $t$ test was used for data analysis 
Table 5 Complications after SASI bypass

\begin{tabular}{lll}
\hline Grade of complication & Type of complication & Number \\
\hline Grade I & Bilious vomiting & $32(5.8 \%)$ \\
& Diarrhea & $15(2.7 \%)$ \\
Grade II & Stoma ulcers & $3(0.54 \%)$ \\
Grade III & Staple line bleeding & $1(0.18 \%)$ \\
& Intestinal obstruction & $1(0.18 \%)$ \\
& Ileal perforation & $1(0.18 \%)$ \\
Grade IV & Calcular obstructive jaundice & $2(0.36 \%)$ \\
& Pulmonary embolism & $1(0.18 \%)$ \\
\hline
\end{tabular}

The current multicenter study was developed to evaluate the efficacy of the SASI bypass as a bariatric and metabolic procedure on a large scale including multiple countries to examine the generalizability of the outcomes of the procedure. More than 500 patients from seven countries were included into this study. Approximately $70 \%$ of patients were female, in line with the female predominance in bariatric surgery as reported in the literature [15].

The \%EWL at 12 months after the SASI bypass was approximately $64 \%$. This was higher than the average $\% \mathrm{EWL}$ after sleeve gastrectomy $(56 \%)$ but less than the average $\%$ EWL after RYGB (68\%) [18]. However, the first study [11] on the efficacy of the SASI bypass reported a much higher \%EWL at 12 months postoperatively, reaching up to $90 \%$. This discrepancy may be explained by the different levels of experience of the operating surgeons. The present study included several centers and different surgeons with varying level of experience with the procedure.

A wide variation in \%EWL after the SASI bypass was also noted in this study. Since the SASI bypass is a fairly new procedure with lack of standardization, this wide range of variability was anticipated. It is expected to decrease as the procedure gains more popularity and gets better standardization. Another factor that may explain this wide variation in the $\% \mathrm{EWL}$ was the wide variation in preoperative BMI $\left(35-80 \mathrm{~kg} / \mathrm{m}^{2}\right)$ which may result in disparate rates of weight loss after surgery.

Predictors for higher \%EWL after the SASI bypass were lower preoperative BMI and lower body weight. It is worth noting that higher preoperative BMIs $\left(>50 \mathrm{~kg} / \mathrm{m}^{2}\right)$ have been reported to be associated with poorer outcomes and lower $\%$ EWL after sleeve gastrectomy [16]. Performing the SASI as a rescue procedure after sleeve gastrectomy was also associated with significantly lower \%EWL (40.9\% vs $63.9 \%$ ) compared with performing the SASI bypass as a primary bariatric procedure. Although weight regain was not observed in patients who underwent the SASI bypass, compared with more than $5 \%$ of patients who undergo sleeve gastrectomy [19], no firm conclusions on weight regain after the SASI bypass can be made owing to the short follow-up of this study.
The main advantage of the SASI bypass was the remarkable remission or improvement in the glycemic state of patients with T2DM. The first published report on the SASI bypass documented normal blood glucose in all patients with T2DM at 3 months postoperatively [11]. This excellent outcome was also demonstrated in the present study as approximately $99 \%$ of diabetic patients showed complete remission or improvement in their diabetic status. These results were close to the incidence of remission of T2DM reported after the duodenal switch $(98.9 \%)$ [20] and higher than that reported after the sleeve gastrectomy $(72-81.6 \%)[15,21]$.

The significant reduction of fasting blood glucose and $\mathrm{HbA} 1 \mathrm{C}$ at 12 months after the SASI bypass demonstrated the therapeutic impact of the procedure on T2DM. Possible mechanisms responsible for the remission of T2DM after the SASI bypass include the restriction of the gastric volume which results in reduction in the caloric intake, the rapid delivery of undigested gastric content into the ileum which has been shown to amplify the nutritive stimulation of the distal gut, and the diminishing of the overstimulation of the proximal gut by having a smaller portion of the meal emptying through the duodenum [11].

Furthermore, the SASI bypass was associated with significant improvements in other obesity-associated comorbidities, particularly GERD. GERD represents a serious challenge for the purely restrictive bariatric procedures such as the sleeve gastrectomy. The increased intra-gastric pressure seen with the sleeve results in a higher incidence of GERD and possible consequences such as Barrett's esophagus [22]. A recent meta-analysis concluded that sleeve gastrectomy may expose the distal esophagus to severe reflux, with an incidence of de novo reflux seen in up to $23 \%$ of patients $[17,23]$.

In contrast, in the present study, more than $90 \%$ of patients with GERD reported improvement in their symptoms after the SASI bypass, whereas no patient complained of de novo GERD postoperatively. This observation may be explained by the impact of adding an anastomosis between the distal gastric sleeve and the ileum which may reduce the intragastric pressure, thus contributing to the amelioration of the symptoms of GERD. It is worth highlighting that $10 \%$ of patients in the present study underwent the SASI as a rescue surgery to correct weight regain or severe GERD after the sleeve gastrectomy.

Regarding the change in nutritional status after SASI bypass, although the reduction in serum albumin levels was significant on the statistical level, it may not be clinically significant since serum albumin levels were within the normal laboratory range indicating that none of the patients developed protein malabsorption after the SASI bypass. A similar decrease in serum albumin level after the SASI bypass was reported in a recent study [24]. On the other hand, Mahdy et al. [11] demonstrated that vitamin D levels actually showed a significant increase at 1 year after the SASI bypass. This was likely due to the systemic 
administration of vitamin $\mathrm{D}_{3}$ prescribed for all patients after the SASI bypass. It is important to note that even purely restrictive procedures can be associated with nutritional deficiencies. The median deficiency rates of iron, zinc, vitamin $\mathrm{D}$, and vitamin $\mathrm{B}_{12}$ after sleeve gastrectomy were reported to be $9 \%, 20 \%$, $35.5 \%$, and $11.7 \%$, respectively [25].

The complication rate of the SASI bypass was approximately $10 \%$, slightly higher than the mean overall complication rate after sleeve gastrectomy $(8.7 \%)$ [15]. However, the vast majority of morbidities after SASI bypass were minor, graded as grade I or II on the Clavien-Dindo scale. The most frequent complication after the SASI bypass was bilious vomiting. Bile reflux seems to be a common phenomenon in patients with a single anastomosis between the gastric pouch and the intestine as it also occurs after the one anastomosis gastric bypass procedure and it has even been reported after RYGB [25-27]. Two patients developed calcular obstructive jaundice caused by de novo gallstone formation after the SASI bypass. Both patients were treated with ERCP and laparoscopic cholecystectomy. One advantage of the SASI bypass, compared with the one anastomosis gastric bypass, is the ability to easily access the biliary system to deal with biliary complications in the future.

Limitations of the present study include its retrospective nature, being associated with inherent risk of selection bias, and the lack of a control group. The varying level of experience of the participating surgeons with the procedure could be another possible limitation. However, the multicenter involvement can also reflect the real-life outcome of the procedure. Another limitation of this study was the short follow-up of 12 months. It is possible that with longer follow-up, there could be significant weight regain, relapse of the comorbid conditions, or significant nutritional deficiencies. We intend to publish the 5-year outcome of the SASI bypass in another future study once the number of patients who completed 5 years of follow-up has been deemed sufficient. Lastly, other nutritional parameters such as serum calcium, zinc, and vitamin $B_{1}, B_{6}$, and $\mathrm{B}_{12}$ were not assessed.

\section{Conclusion}

The SASI bypass is an effective bariatric and metabolic surgical procedure that achieves satisfactory weight loss with remarkable improvement in obesity-related complications, namely T2DM and GERD. The procedure is also associated with a good safety profile with complication rates of approximately $10 \%$, and most of which were minor complications. Randomized trials comparing the SASI bypass with other commonly performed procedures as the sleeve gastrectomy and the RYGB are needed to establish which procedure is superior.
Authors' Contributions Tarek Mahdy designed the study and participated in data collection, drafting, and revision of the manuscript. Sameh Emile and Amr Madyan conducted data analysis and wrote and revised the manuscript. Carl Scho, Abdulwahid Alwahidi, Rui Ribier, Alaa Elsewefy, Martin Busing, Mohammed AL-Haifi, and Emad Sali performed the procedures, followed the patients, collected the data, and revised the final manuscript. Scott Shikora participated in drafting and critical supervision of the manuscript.

\section{Compliance with Ethical Standards}

Conflict of Interest The authors declare that they have no conflict of interest.

Statement of Informed Consent Informed consent was not required for this type of studies (retrospective review of data).

Statement of Human and Animal Rights This study was conducted in accordance with the declaration of Helsinki. Ethical approval for the study was obtained from the Institutional Ethics Committee.

\section{References}

1. Chen L, Magliano DJ, Zimmet PZ. The worldwide epidemiology of type 2 diabetes mellitus present and future perspectives. Nat Rev Endocrinol. 2011;8(4):228-36.

2. Schauer PR, Kashyap SR, Wolski K, et al. Bariatric surgery versus intensive medical therapy in obese patients with diabetes. N Engl J Med. 2012;366:1567-85.

3. Favretti F, Cadiere GB, Segato G, et al. Laparoscopic adjustable silicone gastric banding (Lap-Band): how to avoid complications. Obes Surg. 1997;7(4):352-8.

4. Spak E, Björklund P, Helander H, et al. Changes in the mucosa of the Roux-limb after gastric bypass surgery. Histopathology. 2010;57(5):680.

5. Santoro S, Milleo FQ, Malzoni CE, et al. Entero hormonal changes after digestive adaptation five-year results of a surgical proposal to treat obesity and associated diseases. Obes Surg. 2008;18(1):17-26.

6. Santoro S, Velhote MCP, Malzoni CE, et al. Digestive adaptation: a new surgical proposal to treat obesity based in physiology and evolution. Einstein. 2003;1(2):99-104.

7. Santoro S, Castro LC, Velhote MC, et al. Sleeve gastrectomy with transit bipartition: a potent intervention for metabolic syndrome and obesity. Ann Surg. 2012;256(1):104-10.

8. Angevine KR, Wuescher LM, Andrews K, et al. Menin and GIP are inversely regulated by food intake and diet via PI3/AKT signaling in the proximal duodenum. Nutr Diabetes. 2012;2(12):55.

9. Higashimoto Y, Opara EC, Liddle RA. Dietary regulation of glucose-dependent insulinotropic peptide (GIP) gene expression in rat small intestine. Comp Biochem Physiol C Pharmacol Toxicol Endocrinol. 1995;110(2):207-14.

10. Carr RD, Larsen MO, Winzell MS, et al. Incretin and islet hormonal responses to fat and protein ingestion in healthy men. Am J Physiol Endocrinol Metab. 2008;295(4):779-84. https://doi.org/10.1152/ ajpendo.90233.2008.

11. Mahdy T, Al Wahedi A, Schou C. Efficacy of single anastomosis sleeve ileal (SASI) bypass for type-2 diabetic morbid obese patients: Gastric bipartition, a novel metabolic surgery procedure: A retrospective cohort study. Int J Surg. 2016;34:28-34. https://doi. org/10.1016/j.ijsu.2016.08.018. 
12. Chambers AP, Sandoval DA, Seeley RJ. Integration of satiety signals by the central nervous system. Curr Biol. 2013;23(9):379-88. https://doi.org/10.1016/j.cub.2013.03.020.

13. Lustig RH. The neuroendocrinology of obesity. Endocrinol Metab Clin North Am. 2001;30(3):765-85.

14. Brethauer SA, Kim J, El Chaar M, et al. Standardized outcomes reporting in metabolic and bariatric surgery. Obes Surg. 2015;25(4): 587-606. https://doi.org/10.1007/s11695-015-1645-3.

15. Emile SH, Elfeki H, Elalfy K, et al. Laparoscopic sleeve gastrectomy then and now: an updated systematic review of the progress and short-term outcomes over the last 5 years. Surg Laparosc Endosc Percutan Tech. 2017;27(5):307-17. https://doi.org/10.1097/SLE. 0000000000000418.

16. Elbanna H, Ghnnam W, Negm A, et al. Impact of preoperative body mass index on the final outcome after laparoscopic sleeve gastrectomy for morbid obesity. Ulus Cerrahi Derg. 2016;32(4):238-43. https://doi.org/10.5152/UCD.2016.3275.

17. Yeung KTD, Penney N, Ashrafian L, et al. Does sleeve gastrectomy expose the distal esophagus to severe reflux?: a systematic review and meta-analysis. Ann Surg. 2019; https://doi.org/10.1097/SLA. 0000000000003275. [Ahead of print].

18. Fischer L1, Hildebrandt C, Bruckner T, et al. Excessive weight loss after sleeve gastrectomy: a systematic review. Obes Surg. 2012; 22(5):721-731. doi: 10.1007/s11695-012-0616-1.

19. Lauti M, Kularatna M, Hill AG, et al. Weight regain following sleeve gastrectomy-a systematic review. Obes Surg. 2016;26(6): 1326-34. https://doi.org/10.1007/s11695-016-2152-x.

20. Scopinaro N, Marinari GM, Camerini GB, et al. Specific effects of biliopancreatic diversion on the major components of metabolic syndrome: a long-term follow-up study. Diabetes Care. 2005;28: 2406-11.
21. Sista F, Abruzzese V, Clementi M, et al. Resolution of type 2 diabetes after sleeve gastrectomy: a 2-step hypothesis. Surg Obes Relat Dis. 2018;14(3):284-90. https://doi.org/10.1016/j.soard.2017.12. 009.

22. Stenard F, Iannelli A. Laparoscopic sleeve gastrectomy and gastroesophageal reflux. World J Gastroenterol. 2015;21(36):10348-57. https://doi.org/10.3748/wjg.v21.i36.10348.

23. Emile SH. Gastroesophageal reflux disease after sleeve gastrectomy: the need to predict its onset and prevent its consequences. Obes Surg. 2019;29(8):2625-6. https://doi.org/10.1007/s11695-01903955-9.

24. Salama TMS, Sabry K, Ghamrini YE. Single anastomosis sleeve ileal bypass: new step in the evolution of bariatric surgeries. J Investig Surg. 2017;30(5):291-6. https://doi.org/10.1080/ 08941939.2016.1241841.

25. Emile SH, Elfeki H. Nutritional deficiency after sleeve gastrectomy: a comprehensive literature review. EMJ Gastroenterol. 2017;6(1):99-105.

26. Swartz DE, Mobley E, Felix EL. Bile reflux after Roux-en-Y gastric bypass: an unrecognized cause of postoperative pain. Surg Obes Relat Dis. 2009;5(1):27-30. https://doi.org/10.1016/j.soard.2008. 10.009 .

27. Saarinen T, Räsänen J, Salo J, et al. Bile reflux scintigraphy after mini-gastric bypass. Obes Surg. 2017;27(8):2083-9. https://doi. org/10.1007/s11695-017-2608-7.

Publisher's Note Springer Nature remains neutral with regard to jurisdictional claims in published maps and institutional affiliations. 